\title{
Mitochondrial genetics of amphipods: revealing mechanisms of diversity
}

\author{
Elena V. Romanova \\ Laboratory of Molecular Systematics \\ LIN SB RAS, Irkutsk, Russia \\ elena_romanova@lin.irk.ru \\ Maria D. Logacheva \\ Moscow State University \\ Moscow, Russia, IITP RAS \\ Moscow, Russia
}

\author{
Yurij S. Bukin \\ Laboratory of Molecular Systematics \\ LIN SB RAS, Faculty of Biology and \\ Soil Studies Irkutsk State University \\ Irkutsk, Russia
}

Elena A. Sirotinina

Laboratory of Molecular Systematics LIN SB RAS

Irkutsk, Russia

Kirill V. Mikhailov

Moscow State University

Moscow, Russia

IITP RAS

Moscow, Russia
Vladimir V. Aleoshin

Moscow State University

Moscow, Russia

IITP RAS

Moscow, Russia

Dmitry Yu. Sherbakov

Laboratory of Molecular Systematics

LIN SB RAS, Irkutsk, Russia

Faculty of Biology and Soil Studies

Irkutsk State University

Irkutsk, Russia

\begin{abstract}
We performed a thorough analysis of mitochondrial (mt) genomes of amphipods with the focus on endemic Baikalian species. Possible causes and mechanisms explaining an unusually high variability in $\mathrm{mt}$ genomes of Baikalian amphipods in comparison to that of non-Baikalian species are discussed.
\end{abstract} Baikal

Keywords - mitochondrial genomes, amphipods, Lake Motivation and aim

\section{Motivation}

Mitochondrial (mt) genome sequences are widely used in evolutionary and phylogenetic studies. The architecture of mt genomes (i.e. their shape, length, gene order) can significantly vary in different organisms' groups [1]. The studying of the lineages with different mt genome architecture can help to reveal causes and mechanisms leading to such diversity.

Aim

In our previous study, we sequenced mt genomes of nine endemic amphipod (Crustacea) species from Lake Baikal [2]. We further sequenced mt genomes of Holarctic species Gammarus lacustris and pelagic Baikalian species Macrohectopus branickii. Amphipods is the most numerous and diverse group of Baikalian animals [3]. Genetic studies showed that modern Baikalian amphipod diversity (about 350 species) in the lake appeared by means of adaptive radiation of gammaroidean ancestors which had two independent invasions in the lake $[3,4,5]$. We showed that $\mathrm{mt}$ genomes of Baikalian amphipods significantly vary in length, gene order and gene number in comparison to the modern amphipods of gen. Gammarus, which are their nearest relatives. Thus we applied different bioinformatics approaches to discover the mechanisms of $\mathrm{mt}$ genome architecture diversity in Baikalian amphipods.

\section{Methods}

We had performed phylogenetic inference of amphipod species with available mt genomes (100 species in total) based on amino acid sequences of their mt protein-coding genes [6]. The scenarios of $\mathrm{mt}$ gene order rearrangements were estimated using CREx [7]. The prediction of tRNA genes was made by MitFi [8]. For this analysis, we developed amphipod-specific models which showed a better performance than the default metazoan models implemented in MitFi. The non-coding parts of amphipod $\mathrm{mt}$ genomes were tested to contain repeated sequences and open reading frames. We used a custom $\mathrm{R}$ script to assess rates of molecular evolution in Baikalian amphipods and the group of gen. Gammarus.

\section{Results}

Phylogenetic analysis allowed us to deduce an ancestral mt gene pattern for both lineages of Baikalian amphipods, which appeared to be the same as one for species of gen. Gammarus group. Baikalian species show a drastic diversity in their mt genome lengths (from 14370 to 42256 bp.). A pelagic species $M$. branickii has the longest $\mathrm{mt}$ genome. The largest part of this genome consists of the numerous repetitive non-coding regions, which, presumably arose due to the proliferation of ribosomal genes. Mt genome sequences of seven out of twelve Baikalian amphipods species possess from one to four additional tRNA genes. Four species have tRNA genes that underwent to remolding (changing tRNA gene identity through a mutation in their anticodon sequences) [9, 10]. Among 88 non-Baikalian amphipod species, only five species have additional tRNA genes in their mt genomes and four of them have tRNA remolding cases. The frequent changes of gene number and order as well as other "peculiarities" in $\mathrm{mt}$ genomes of Baikalian amphipods in comparison to species of gen. Gammarus may be explained by the frequent sequence duplication and loss events happening in the former group. Thus we may assume that the specific environmental conditions in Lake Baikal allow the appearance and maintenance of such $\mathrm{mt}$ genomes without loss of species fitness.

\section{Acknowledgment}

The work was supported by the governmentally funded project 0345e2019e0004 (AAAA-A16-116122110060-9). 


\section{References}

[1] D.V. Lavrov and W. Pett, "Animal mitochondrial DNA as we do not know it: mt-genome organization and evolution in nonbilaterian lineages, " Genome Biol. Evol., vol. 8, pp. 28962913, 2016.

[2] E.V. Romanova, V.V. Aleoshin, R.M. Kamaltynov, K.V. Mikhailov, M.D. Logacheva, E.A. Sirotinina et al. "Evolution of mitochondrial genomes in Baikalian amphipods", BMC genomics, vol. 17, pp. 1016, 2016

[3] R.M. Kamaltynov, "Amphipoda: Gammaroidea in Angara and Yenisei rivers," in Index of animal species inhabiting Lake Baikal and its catchment area, Vol. 2, O.A.Timoshkin, V.I. Proviz, T.Y. Sitnikova, Z.V. Slugina, N.G. Melnik, Eds. Novosibirsk: Nauka, 2009, pp. 297-329.

[4] K.S. Macdonald Iii, L. Yampolsky, J.E. Duffy, "Molecular and morphological evolution of the amphipod radiation of Lake Baikal,” Mol. Phylogenet. Evol., vol. 35, pp. 323-343, 2005.

[5] S.A. Naumenko, M.D. Logacheva, N.V. Popova, A.V. Klepikova, A.A. Penin, G.A. Bazykin et al., "Transcriptomebased phylogeny of endemic Lake Baikal amphipod species flock: fast speciation accompanied by frequent episodes of positive selection," Mol. Ecol., vol. 26, pp. 536-553, 2017.
[6] L.T. Nguyen, H.A. Schmidt, A. von Haeseler, B.Q. Minh, "IQTREE: a fast and effective stochastic algorithm for estimating maximum-likelihood phylogenies," Mol. Boil. Evol., vol. 32, pp. 268-274, 2014.

[7] M. Bernt, D. Merkle, K. Ramsch, G. Fritzsch, M. Perseke, D. Bernhard et al., "CREx: inferring genomic rearrangements based on common intervals," Bioinformatics, vol. 23, pp. 29572958, 2007.

[8] F. Jühling, J. Pütz, M. Bernt, A. Donath, M. Middendorf, C. Florentz, et al., "Improved systematic tRNA gene annotation allows new insights into the evolution of mitochondrial tRNA structures and into the mechanisms of mitochondrial genome rearrangements," Nucleic Acids Res., vol. 40, pp. 2833-2845, 2012.

[9] P. Cantatore, M.N. Gadaleta, M. Roberti, C. Saccone, A. C. Wilson, "Duplication and remoulding of tRNA genes during the evolutionary rearrangement of mitochondrial genomes," Nature, vol. 329, pp. 853-855, 1987.

[10] E.V. Romanova, Y.S. Bukin, K.V. Mikhailov, M.D. Logacheva, V.V. Aleoshin, D.Y. Sherbakov, "Hidden cases of tRNA gene duplication and remolding in mitochondrial genomes of amphipods," Mol. Phylogenet. Evol. vol. 144, pp. 106710, 2020. 